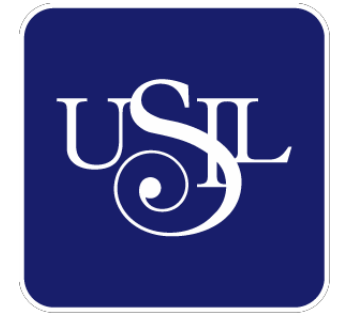

UNIVERSIDAD

SAN IGNACIO

DE LOYOLA

FACULTAD DE HUMANIDADES

Carrera de Psicología

\title{
ESTILOS DE LIDERAZGO DE LOS DIRECTIVOS Y SATISFACCIÓN LABORAL DEL PERSONAL ADMINISTRATIVO EN UN INSTITUTO SUPERIOR
}

Tesis para optar el Título Profesional de Licenciado en Psicología

VIRGINIA GIOVANA ROSALES ANDAGUA

(0000-0003-1270-434X)

\section{FABIANA TRUJILLO VERA}

(0000-0003-1192-9176)

Asesor:

Mg. Jhonatan Steeven Baruch Navarro Loli

(0000-0001-6264-3157)

Lima - Perú

2020 


\section{Contenido}

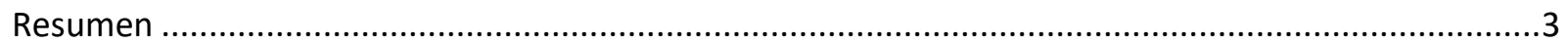

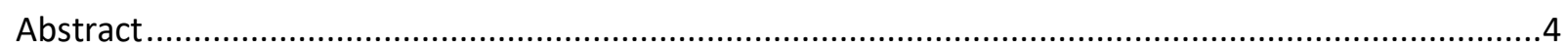

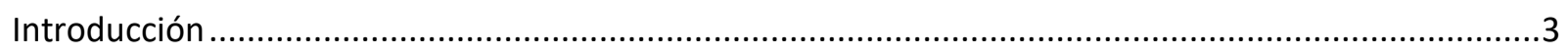

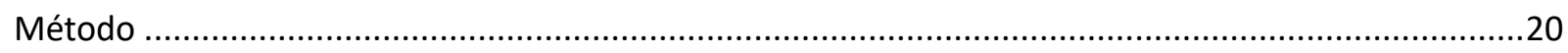

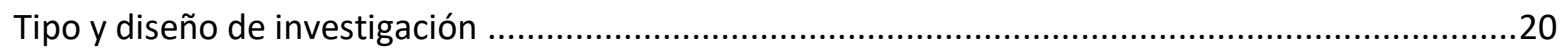

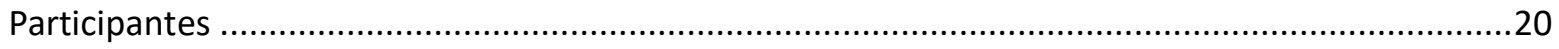

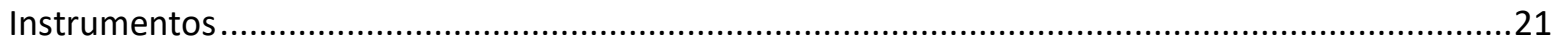

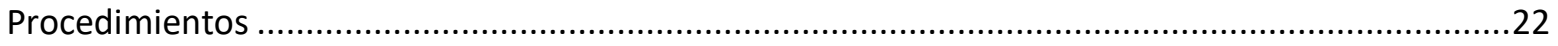

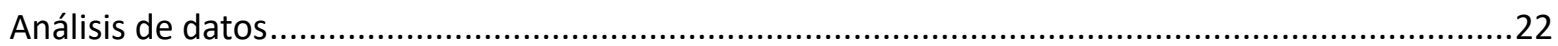

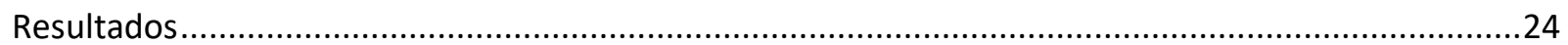

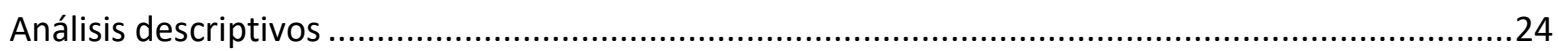

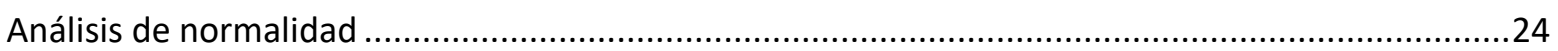

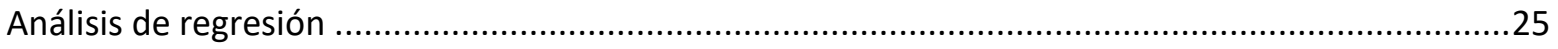

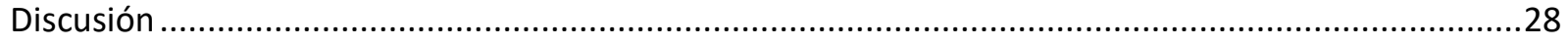

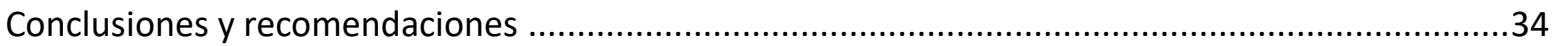

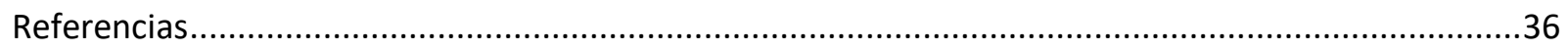

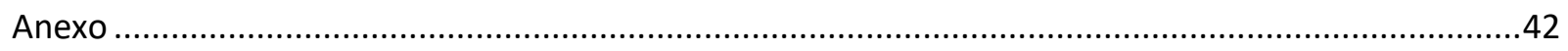

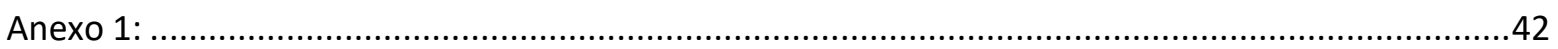

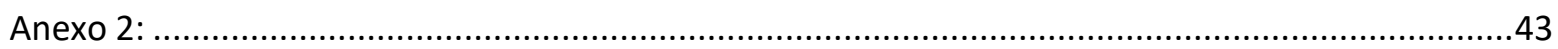

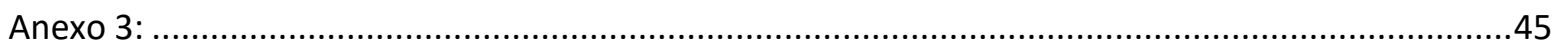




\section{Resumen}

La presente investigación tiene como objetivo a identificar si los Estilos de Liderazgo (EL) de los directivos predicen la Satisfacción Laboral (SL) del personal administrativo en un instituto de Educación Superior. El estudio se realizó con una muestra de 84 trabajadores con edades comprendidas entre 21 a 71 años $(\mathrm{M}=38.1)$. Para el análisis de la variable EL se empleó el Cuestionario de EL CELID forma S y para $S L$ se empleó el Cuestionario de Satisfacción S21/26. Los resultados indican que el Estilo de Liderazgo Transformacional (LTF) predice de manera positiva y significativa las dimensiones de la $S L$ y el Estilo de Liderazgo Transaccional (LT) predice de manera positiva y significativa únicamente la dimensión Satisfacción con la participación (SP). Se concluye que el estilo con mayor impacto positivo en la SL es el estilo LTF, por ende, se recomienda a las instituciones instaurar en el perfil de los directivos este estilo.

Palabras claves: Satisfacción Laboral, Estilos de Liderazgo, Trabajadores 


\begin{abstract}
The present research aims to identify if the leadership styles of managers predict the job satisfaction of administrative staff in a higher education institute. The study was conducted with a sample of 84 workers aged between 21 and 71 years $(M=38.1)$. For the analysis of the Leadership Styles variable, the CELID Leadership Styles Questionnaire was used S form and for Job Satisfaction, the S21/26 Satisfaction Questionnaire was used. The results indicate that the Transformational Leadership Style predicts in a positive and meaningful way the dimensions of Job Satisfaction and the Transactional Leadership Style predicts in a positive and meaningful way only the dimension Satisfaction with the participation. It is concluded that the style with the greatest positive impact on job satisfaction is the transformational style, therefore, it is recommended that institutions establish this style in the profile of managers.
\end{abstract}

Keywords: job satisfaction, leadership styles, workers 


\section{Introducción}

En el 2019 la Organización Mundial de la Salud (OMS) notificó la aparición del COVID19 en la ciudad de Wuhan, China y se propago rápidamente en distintos países del mundo, lo cual desencadeno diversos cambios a nivel social, económico y educativo. Así mismo, en Latinoamérica, a nivel social, las cifras de desempleados superan los 49.7 millones de personas generando efectos graves en la pobreza y desigualdad; a nivel educativo, cerca de seis millones de niños y jóvenes se ven imposibilitados de estudiar a distancia debido a la carencia de medios tecnológicos; y finalmente, a nivel económico el impacto fue mayor, porque éste disminuyó su crecimiento en 5.3\%, generando la caída de la inversión privada, cierre de más de 2 millones de empresas y desencadenando el desempleo (Comisión Económica para América Latina y el Caribe [CEPAL], 2020).

A mediados de marzo, el Perú se declaró en estado de emergencia, a través del Decreto Supremo No 010-2020-TR, en consecuencia, la paralización de distintos sectores económicos que ocasionaron grandes cambios en las condiciones laborales, la salud y seguridad del personal. Por ejemplo, según cifras del Ministerio de Trabajo y Promoción del Empleo (MINTRA, 2020), aproximadamente 220,000 trabajadores formales pasaron a realizar trabajo remoto, desencadenando en muchos casos el incremento de la jornada laboral y la falta de equilibrio entre la vida familiar y laboral. En el caso de puestos laborales que por su naturaleza no pueden realizar trabajo remoto se ajustaron a medidas como; reducción de salario y jornada laboral, licencia sin goce de haber, goce y adelanto de vacaciones y la suspensión perfecta de labores y en el peor de los casos fueron despedidos. Debido a esto el Instituto Nacional de Estadística e 
Informática (INEI, 2020), informó el incremento en más de 8.8\% la tasa del desempleo, generando grandes pérdidas económicas, personales y empresariales.

Como consecuencia de esta crisis global, la economía de muchas empresas resultó afectada, desencadenando la disminución salarial, suspensión perfecta de labores y reducción de personal, lo que llevo a los directivos a identificar mejores estrategias para motivar y comprometer a sus equipos basándose en motivadores intrínsecos (Barajas, Betancur, Lopez. \& Rodríguez, 2020). En ese sentido, el liderazgo se posiciona como una de las competencias más demandadas para el logro de los objetivos y retos organizacionales, ya que impacta en la satisfacción laboral y productividad del equipo (Carhuachin, 2019).

Por lo expuesto en el párrafo anterior, las empresas deberán de reforzar en sus procesos la contratación de perfiles con habilidades de liderazgo, ya que este está ligado o es considerado como una de las bases para el éxito organizacional (Almiron, Trejo, \& Garcia, 2015). Así mismo, cabe señalar que un líder difiere de un directivo; el primero busca el logro de los objetivos basados en valores, ideales e intercambios emocionales. Mientras que el directivo enfoca su energía en el uso de criterios racionales como la estabilidad, orden y eficiencia (Almudena \& Pastor, 2010).

Por su parte Castillo, Medina, Bernardo, Reyes y Ayala (2019) al investigar la variable liderazgo, encontraron que, al emplear los estilos de LTF y LT, el clima organizacional saludable se incrementa. Del mismo modo, Berdecía, Gonzales y Carrasquillo (2013) afirmaron que los estilos de estilos de LTF seguido del LT aportan al éxito organizacional; y el estilo LTF es considerado como uno de los estilos más adecuados que guían a las empresas a la innovación y creatividad, además está asociado de manera positiva con la satisfacción, efectividad percibida 
con el líder y motivación para realizar horas extras. (Álaval et al, 2016; Hermosilla, Amutuo, Da Costa \& Paez, 2016).

Por otro lado, Escandon y Hurtado (2016) indican que el estilo de LF o pasivo evitativo se asocian de manera negativa con el desempeño de los trabajadores y el éxito organizacional, y que los resultados pueden ser poco alentadores. Además, Vignoli, Mariani, Guglielmi y Violante (2018) evidencian que el estilo LF influye de manera negativa en el aprendizaje de los miembros del equipo, ya que existe poco interés por parte del líder en la tarea y supervisión para transferir conocimientos.

La SL, es la percepción que tienen los trabajadores sobre los factores intrínsecos como el puesto de trabajo, motivación por el aprendizaje, desarrollo de línea de carrera, etc; y los factores extrínsecos tales como el salario, infraestructura, beneficios remunerativos, clima organizacional, cultura organizacional, etc., que prescriben su bienestar y compromiso con su desempeño y productividad laboral (Chiang, Gómez, \& Hidalgo, 2017). Por otro lado, la SL genera una relación afectiva o estado emocional hacia el trabajo que se refleja en el gusto por lo que hace (Anaya \& López, 2015; Cernas, Mercado, \& Davis, 2018; González et al., 2014; Locke, 1969; Lupano \& Castro, 2018; Rodríguez et al., 2011).

Gamero (2013) indica que la felicidad resulta ser clave para generar SL, además Sanin y Salanova (2013) mencionan que la SL predice comportamientos de flexibilidad y habilidades efectivas de adaptación al cambio, mientras que, las personas insatisfechas experimentan los cambios de manera cerrada y con irritación. También se ha mencionado que las personas que tienen niveles altos de satisfacción son aquellas que tienen mayor intención de permanecer en la empresa (Amarillo \& Mosquera, 2012; Ziebell, Natividad, Soares, \& Sandoval, 2019). De la misma manera, otros estudios concluyen el clima organizacional está asociado a los niveles de 
SL, y desempeño organizacional, es decir, aquellos trabajadores que se encuentren satisfechos con el trabajo, la relación con sus jefes, y satisfacción con el reconocimiento reflejan mayores niveles de productividad y desempeño (Chiang \& Ojeda, 2011; Montoya, et al., 2017; Pedraza, 2020).

En la actualidad existen estudios que correlacionan los EL con la SL, es así que un estudio dirigido por Gallegos y Miranda (2019) concluyeron que los EL predicen de manera significativa las dimensiones de la SL. Del mismo modo, Valencia (2019) encontró que los EL del equipo directivo presentan una relación positiva con la SL de los docentes.

Por su parte Chiang, Gómez y Salazar (2014), indican que el LTF fomenta la satisfacción del trabajador. Otros autores como Guerrero (2016), indican que los EL que adoptan los líderes de las organizaciones, llegan a influir en la SL de los trabajadores, así como en el compromiso que ellos tienen hacia la organización. En ese sentido el estilo de LTF inspira a sus trabajadores para alcanzar esfuerzo y dedicación; sin embargo, el LT busca motivarlos a través de refuerzos, llegando a correlacionar de manera positiva con el esfuerzo extra (Álaval et al., 2016) Finalmente, otro estudio realizado por Perilla y Gómez (2017) concluyó que el estilo de LTF influye de manera positiva en la SL y ello significa menor malestar psicológico de los trabajadores.

Finalmente, debido a las condiciones actuales, la importancia de los EL y la SL en el contexto organizacionales es fundamental para el crecimiento, adaptación y éxito de las organizaciones, es así que la presente investigación formula la siguiente pregunta: ¿Determinar si los EL predicen la SL en el personal administrativo de un Instituto de Educación Superior de Lima y Arequipa? 


\section{Marco teórico}

El liderazgo desde la perspectiva de Bass (1990) es considerada como un proceso de interacción entre los integrantes de un grupo, y que el líder es quien guía el logro de los objetivos, resuelve problemas, creando para ello objetivos en común. Por su parte Chiavenato (2002) y Jiménez y Villanueva (2018) consideran el liderazgo como un pilar clave para conseguir objetivos, metas y aspiraciones, que orientan el trabajo colaborativo de calidad, además el líder emplea la comunicación como una herramienta de influencia en sus seguidores y grupal. Así mismo, Ayuso (2017), caracteriza a los líderes de hoy como agentes de cambio organizacional, y fomenta el trabajo colaborativo procurando el desarrollo de los trabajadores y la organización. Cabe mencionar que el liderazgo no solo incluye al líder, sino que además considera otros elementos como los seguidores, supervisores, contexto, compañeros y la cultura (Avolio, 2007; Avolio, Walumbwa, \& Weber, 2009).

El presente estudio emplea el modelo de rango completo de Bass y Avolio (1996) que contempla tres EL que son: LTF, LT y Laissez Faire (LF).

El $L T F$, es la capacidad que tiene el líder para motivar e inspirar a los seguidores con la finalidad de convertirlos en personas productivas capaces de lograr resultados deseados que vayan más allá de sus beneficios personales (Bass, 1985, 1990; Burns, 1978; Bass \& Avolio, 1994, 2006). Este estilo se caracteriza por motivar las expectativas, desarrollar ideas y enfoques; así como mostrar responsabilidad, confianza en sus juicios, muestra optimismo, respeto y atención a los seguidores. Además, resulta muy útil para el manejo del cambio organizacional ya que otorgan a sus seguidores libertad para afrontar con éxito situaciones conflictivas, brindándoles apoyo, tolerancia y seguridad (Bass, 1985; Vargas \& Mayne, 2019). 
Del mismo modo, Velásquez (2006), indica que este estilo es un proceso que involucra el trabajo colaborativo, a fin de aumentar la motivación, la moral y el rendimiento de los seguidores, orientado a su vez el logro de los objetivos organizacionales. Así mismo, otros autores lo definen como un agente de cambio que busca obtener los resultados organizacionales a través de la comunicación clara de la visión, el carisma, confianza, inspiración del compromiso y motivación al equipo. Estos líderes emplean el entusiasmo y la energía para inspirar a sus seguidores (Almiron et al., 2015; Hermosilla et al., 2016; Lerma, 2007).

El estilo de $L T$ se basa en el intercambio de favores y promesas entre el líder y los seguidores con la finalidad de coincidir intereses organizacionales y personales. En este estilo, el líder aclara las recompensas que se alcanzarán por el esfuerzo realizado y acciones correctivas en caso que incumpla los estándares deseados (Bass, 1999, 2007; Quispe, 2020; Varela, 2010). Este estilo se caracteriza por un aprendizaje interdependiente de dar y recibir, y cuando la recompensa se extingue, disminuye la motivación (Oliveira \& Ferreira, 2015).

Así mismo, está orientado al intercambio de recompensas entre el líder y seguidores, es decir, el líder adopta un rol activo y motiva a los seguidores a partir de recompensas económicas o refuerzos de otro tipo sujetas al desempeño. Este estilo puede resultar egoísta, ya que ambas partes buscan un propio beneficio, el líder obtiene mayor productividad, mientras que los seguidores recompensas. Cuando este estilo es bien aplicado tiene efectos positivos en el rendimiento y satisfacción de los trabajadores (Fischman, 2005; Molero, Recio \& Cuadrado, 2010).

Finalmente, el estilo de LF o pasivo evitador, es uno de los estilos más negativos, ya que estos líderes evitan tomar decisiones, no reconocen el trabajo de los seguidores y no hacen uso de la autoridad que tienen para el logro de los objetivos organizacionales (Bass \& Riggio, 2006). 
Este estilo tiene poca o nula atención en el desarrollo organizacional y de los seguidores, ya que asume que el líder debe tener un perfil bajo y por ello procura estar lejos de los problemas; y solo será capaz de intervenir cuando un problema se vuelva realmente serio (Molero et al., 2010; Warrick, 1981).

Del mismo modo, se caracteriza por la ausencia de responsabilidad y autoridad para la toma de decisiones de parte del líder (Alejandro \& Casulo, 2004), siendo quienes asumen estas responsabilidades y protagonismo los trabajadores; además existe poco control y motivación de procesos, ocasionando conflictos de rol, justicia procedimental y el acoso psicológico en ambientes laborales (Rodríguez, Gil, \& Moreno, 2012).

Por otro lado, la segunda variable de estudio es la SL. Diversos autores la han definido desde varias perspectivas. Peiró (1984) define la SL como un conjunto de actitudes positivas que el trabajador experimenta frente al trabajo que tiene y su entorno (Meliá et al., 1998). Por su parte Locke (1968) define la SL como un estado emocional y actitudinal positivo o placentero, que resulta a través de la evaluación del gusto hacia la experiencia o el trabajo. De acuerdo a Robbins (2004) la SL se determina mediante recompensas que brindan ciertas tareas, los premios, condiciones laborales, relaciones con el grupo a las que los trabajadores están expuestos. También, indica que los rasgos de personalidad influyen en la percepción que siente el trabajador de su bienestar y agrado con las funciones que desempeña, es así que, cuanto más se ajuste el perfil del puesto a la personalidad del trabajador, éste presentara mayores niveles de satisfacción. Es importante aclarar que la SL está asociado con el desempeño laboral y otros factores como el ausentismo, rotación de personal y conducta cívica organizacional (Bitsch \& Hogberg, 2005). 
Resulta imposible abordar esta variable sin antes mencionar el modelo teórico de Herzberg, con su teoría de factor dual. En ella se habla de dos factores que influyen en la SL de los trabajadores, tales como: Factores motivadores o intrínsecos, que hace referencia a las variables relacionadas con los sentimientos positivos hacia las funciones mismas del trabajo; entre las que destacan los logros, responsabilidades, reconocimiento y promoción; estos factores están mayormente asociados a la satisfacción. Por otro lado, están los fatores de higiene, que se asocian al contexto del trabajo tales como la infraestructura, relaciones interpersonales, salario, liderazgo y políticas de la compañía; estos factores se asocian normalmente a la SL (Herzberg, 1966).

Por otro lado, Meliá et al. (1998) identifican cuatro dimensiones de la SL:

La satisfacción con la supervisión (SS), la cual está orientado al tipo de relación que se pueda desarrollar con los individuos, sean estos, compañeros o líderes. Esto se refiere a la percepción que se tiene sobre el rol del líder en las tareas encomendadas, participación de las decisiones, la evaluación de desempeño y el apoyo o igualdad del trato que recibe. (Meliá et al., 1990). Hace referencia además a las relaciones horizontales con los otros departamentos a fin de promover la comunicación, apoyo, feedback disponible, reconocimiento, amistad y grado de comunicación organizacional (Flores \& Salazar, 2019). Este indicador está relacionado con la insatisfacción, puesto que la mala relación suele ser ocasionada por comportamientos pasivos o agresivos de los compañeros y jefes. (López \& Magallanes, 2017).

La satisfacción con la participación (SP) hace referencia a la formación que reciben por parte de la empresa y por la oportunidad de tener participación en la toma de decisiones del grupo emitiendo opiniones o juicios que aporten a los objetivos del área de trabajo (Meliá et al., 1990; Meliá \& Peiró, 1989). Además, permite usar sus habilidades y autonomía en la toma de 
decisiones, por lo que un reto moderado causa placer y satisfacción (López \& Magallanes, 2017). También se refiere a al trato justo e igualitario que pueda recibir el colaborador dentro de su área o equipo de trabajo. (Ruiz, 2009).

La satisfacción con la calidad de producción (SCP) son un conjunto de sentimientos y percepciones positivos que tiene el colaborador sobre los servicios o resultados que obtiene en el ejercicio de sus funciones (Kellogg, Youngdahl, \& Bowen,1997). Se refiere también a los medios materiales y recursos con los que el colaborador dispone para realizar sus funciones y garantizar la calidad en sus resultados finales (Meliá et al., 1990) Así mismo, incluye los objetivos, metas e indicadores de producción alcanzados de manera individual o dentro del área. (Ruiz, 2009).

La satisfacción intrínseca (SI) que está orientado a la motivación y satisfacción del trabajador con respecto a su autonomía, oportunidades de desarrollo, nuevos retos, logros, responsabilidades y promoción (Meliá et al., 1990). Además, se refiere a las oportunidades de experimentar nuevos retos, metas y objetivos, haciendo lo que le agrada y aquello en que destaca (Meliá \& Peiró, 1989). Por su parte, Bargsted (2019) indica que los trabajadores con mayor nivel de autoeficacia muestran mayores niveles de SL. Del mismo modo, este indicador hace referencia a las necesidades de los trabajadores con aspiraciones de crecimiento profesional y promoción, al notar un estancamiento en su línea de carrera, se produce un desequilibrio entre lo que ella esperaba y lo que ha obtenido realmente, generando insatisfacción y posible abandono laboral (López \& Magallanes, 2017). 


\section{Antecedentes}

Antecedentes nacionales

Gallegos y Miranda (2019) realizaron un estudio para determinar la influencia de los EL en la SL. Su estudio empleó una muestra de 251 trabajadores de diferentes empresas de Lima y Arequipa de edades entre 21 y 65 años. El instrumento de investigación empleado para la variable EL fue la Escala Multifactorial de EL - MLQ-5x (2000) y para la variable SL la Escala de Satisfacción Organizacional 21/26 de Meliá y Peiró (1999). Los resultados de la investigación reflejaron que el Estilo de LTF predice de manera significativa y positivamente las siguientes dimensiones SI $(\beta=.34), \mathrm{SS}(\beta=.44)$ y SCP $(\beta=.40)$. En cuanto a LT predice únicamente de manera significativa y positivamente con las dimensiones de $\mathrm{SP}(\beta=.32)$ y $\mathrm{SCP}$ $(\beta=32)$. Finalmente, el estilo LF no predice de manera significativa ninguna de las dimensiones de la SL. Las conclusiones indican que los estilos de LTF y LT predicen de manera significativa y positivamente algunas dimensiones de la SL, mientras que el LF no predice ninguna de las dimensiones de la SL, eso indica que, si se adopta el estilo de LTF seguido del LT se pueden alcanzar mayores niveles de SL.

Paucar (2013) propuso investigar la relación de los EL del personal directivo y la SL del personal administrativo y docente. Para el estudio se empleó una muestra de 36 personas entre directivos, docentes y administrativos. Para el análisis de las variables los autores crearon un cuestionario basado en los EL de Lewin (1939, citado por Alvarado, 2003; Castaño, 2013; Zuzama, 2015) obteniendo una confiablidad mediante el método de mitades partidas y la validez por el juicio de experto, que exploró ambas variables. Los resultados indicaron que existe relación positiva y significativa fuerte entre los EL y la SL $(r=.767)$. El estilo Autocrático correlaciona de manera inversa significativa muy fuerte, mientras que el permisivo correlaciona 
de manera inversa significativa fuerte. Finalmente, el estilo democrático correlaciona de manera positiva y significativa débil. Los autores concluyen que los directivos de esa institución tienen características no democráticas, actitudes permisivas y conductas autoritarias lo que repercute de manera inversa en la satisfacción de sus trabajadores.

Chacón (2016) mediante un estudio de investigación, se enfocó en analizar la relación entre el Estilo de LTF y la SL. La muestra empleada fueron 65 trabajadores de una empresa privada con edades entre 19 a 58 años del rubro de transporte. Los instrumentos empelados fueron la Escala de LTF de Bass y Avolio (2000) y adaptada a la realidad chilena por Vega y Zavala (2004); para el caso de la Variable SL se empleó la Escala de SL de Yamamoto (2012). Los resultados muestran una correlación significativa y positiva entre las dimensiones de la variable LTF con la variable SL, siendo las dimensiones del LTF tales como consideración individualizada $(\mathrm{r}=.440)$, motivación inspiradora $(\mathrm{r}=.416)$, influencia idealizada $(\mathrm{r}=.526)$ y estimulación intelectual $(\mathrm{r}=.300)$ que se relacionan de manera positiva y significativa con la SL. Al elevarse la percepción de LTF en sus líderes inmediatos, la SL será más elevada. Las conclusiones indican que el LTF se asocia de manera significativa y positiva la SL, eso significa que si los líderes muestran un estilo transformador los subordinados presentaran mejores niveles de SL.

Redolfo (2017) investigó la relación que existe entre LTF y SL. La muestra estuvo conformada por 154 trabajadores de la UGEL 04 - Comas. El instrumento empelado fueron el Cuestionario de LTF - MLQ-5X (2004) y para la variable SL se utilizó el Cuestionario de SL 21/23 de Meliá y Peiró (1998). Los resultados describen una relación positiva y significativa entre LTF y SL $(r=.219)$. Las dimensiones del LTF, tales como estimulación intelectual $(\mathrm{r}=$ .114), motivación inspiracional $(\mathrm{r}=.243)$, consideración individual $(\mathrm{r}=.451)$ y influencia 
idealizada $(\mathrm{r}=.177)$ se relación de manera positiva con la variable SL. Las conclusiones indican que todas las dimensiones del LTF se asocian de manera positiva y significativa, es decir, que a mayor LTF mayor SL.

Pacheco (2019) investigó la relación entre LTF con la SL en trabajadores de una empresa privada. El estudio fue aplicado en 300 trabajadores de una empresa privada con edades entre 24 a 40 años. Los instrumentos empleados fueron la Escala de LTF elaborado por Rafferty y Griffin (2004) y para la variable SL se empleó la Escala de SL (SL-SPC) de Palma (2005). Los resultados indican que no existe correlación estadísticamente significativa entre el LTF y SL $($ rho $=-.037, \mathrm{p}>.05)$, lo cual podría deberse a que el personal estuvo evaluando la conducta de liderazgo mas no al líder, es decir, un líder que no cuenta con un liderazgo especifico o ideal y que este genera un ambiente laboral no adecuado. Las conclusiones indican que no existe correlación significativa entre LTF y SL, así como no existen diferencias significativas según la edad y sexo de los evaluados en cada una de las variables.

Antecedentes internacionales

Franco, Reyes y Cuadrado (2017) analizaron de la incidencia de los EL aplicada a la SL en Ecuador. La muestra empleada fue 322 trabajadores del área administrativa y operativa comprendidas entre edades de 20 y 39 años. Los instrumentos empleados para el análisis de la variable de EL Cuestionario de Multifactorial de Liderazgo (MLQ) forma 5X de (Bass \& Avolio, 2000); mientras que para la variable SL el Cuestionario de Satisfacción 20/23 de Melia y Peiro (1989). Los resultados demuestran que existe una correlación significativa baja entre los estilos LTF $(r=$.273), LT $(r=$.295) y el LF $(r=-.079)$ con la satisfacción en general. Concluyen que el estilo LT seguido del LTF se asocia de manera significativa con la satisfacción laboral y al combinar ambos estilos se pueden implementar estrategias para el alcance de los objetivos. 
Samson (2016) realizó un estudio con la finalidad de investigar la influencia de la relación entre supervisor y empleado, EL y SL percibidos, compromiso y desempeño laboral. La muestra estuvo conformada por 123 empleados en Nigeria, con edades de entre 20 y 56 años. Los instrumentos utilizados para los ELfueron, el Multifactor Leadership Questionnaire (MLQ - Form 5X) (Bass, 1998; Bass \& Avolio, 1994). Para la medición de la SL se utilizó la Escala de SL de (Macdonald \& Maclntyre, 1997). Los resultados muestran que la SL se correlaciona positivamente con el LTF y LT $(r=.71)$, mientras que el estilo LF correlaciona negativamente $(r=-.20)$. Se concluye que los estilos de LTF y LT se asocian de manera positiva e influyen en la SL y en otras variables como el compromiso y relación entre pares y jefes. Por ello, los investigadores recomiendan la implementación de programas para el desarrollo de habilidades de dirección que impactan en el compromiso, satisfacción laboral y relación entre pares.

Ramandeep, Vaishali y Neetu (2016) realizaron una investigación con el objetivo de examinar la relación entre EL y SL. La muestra la conformaron 421 mujeres de puestos superiores en empresas del sector servicio de la India. Los instrumentos empleados para la recolección de datos constaron de tres secciones, es decir, perfil demográfico, elementos sobre diversas dimensiones de los EL y escala de satisfacción de los empleados. Los resultados muestran que las mujeres con estilo de LTF y democrático tienen un impacto positivo en la SL de sus trabajadores, con una correlación de $(\mathrm{r}=.82)$ y $(\mathrm{r}=.42)$ respectivamente. Por su parte las que poseen un estilo de LT y LF tuvieron un efecto negativo en la SL de sus empleados, una correlación $(\mathrm{r}=-.61)$. Se concluye que las mujeres para proporcionar un equipo eficaz y satisfecho deberán adoptar un estilo de LTF o democrático.

Saleem (2015) se propuso investigar los EL en la SL y verificar si las políticas organizacionales percibida tiene un rol mediador. La muestra estuvo conformada por 217 
docentes de nivel universitario de la ciudad de Pakistán. Los instrumentos utilizados fueron el Cuestionario Multifactorial de Bass y Avolio (1997) y la escala de SL de Spector (1997). Los resultados obtenidos indican que los EL se relacionan de manera positiva y moderada con la variable SL $(r=$.638). Los valores resultados indican que la relación entre SL y el LTF fue de $\mathrm{r}=.901$ y con LT fue de $\mathrm{r}=.843$. Se concluye que el estilo de LTF se relaciona de manera positiva con la SL eso quiere decir que el líder inspira a partir de motivadores e induce a cambios de comportamiento de todos los miembros del equipo. Por el contrario, los líderes LT motivan a los subordinados al desarrollo de la organización mediante de premios o castigos, en caso no se cumpla este proceso de dar y recibir podría generar la disminución de la motivación de los miembros de la organización.

Oliva y Molina (2016) realizaron un estudio para determinar los EL de directores y la SL percibida por los funcionarios. La muestra estuvo conformada 171 trabajadores del sector salud de Chillán - Chile. Las técnicas empleadas para la recolección de datos fueron el cuestionario de escala de SL SL/2006/39, la cual fue desarrollado por Chiang, Salazar, Martín y Núñez (2007) en base al instrumento S21/26 (1990) y el S4/82 (1986) de Meliá y Peiró (1989); en el caso de los EL se empleó y cuestionario de liderazgo situacional, publicado por Chiang, Salazar y Nuñez (2007). Los resultados indicaron que existe una relación positiva moderada entre el Estilo de Liderazgo Directivo $(r=.558)$, Estilo de Liderazgo Persuasivo $(r=.549)$ y Estilo de Liderazgo Participativo $(r=.483)$ con la SL, resaltando el Estilo de Liderazgo directivo con una mayor asociación. Sin embargo, el estilo de Liderazgo Delegativo, presentó una relación débil y positiva con la SL ( $\mathrm{r}=.112$ ). Por lo tanto, se confirma que los EL influyen de manera positiva y moderada SL, sin embargo, no es la única variable que influye en la SL, pero se convierte en la más relevante para la dinámica organizacional. 
De acuerdo a lo expuesto, se formula la siguiente pregunta de investigación ¿Los EL predicen la SL del personal administrativo de una Institución Educativa Superior de Lima y Arequipa? Así mismo, en cuanto a los objetivos específicos, se propone lo siguiente:

- Identificar si el estilo de LTF es predictor de las dimensiones de SL en el personal administrativo de un Instituto de Educación Superior de Lima y Arequipa.

- Identificar si el estilo de LT es predictor de las dimensiones de SL en el personal administrativo de un Instituto de Educación Superior de Lima y Arequipa.

- Identificar si el estilo de liderazgo LF es predictor de las dimensiones de SL en el personal administrativo de un Instituto de Educación Superior de Lima y Arequipa.

La investigación se justifica de forma teórica, ya que permite actualizar la bibliografía respecto a las variables EL y SL en el sector educativo. Esto es importante porque hay investigaciones donde se reportan que los EL son predictores de la SL (Paucar, 2013; Gallegos \& Miranda, 2019; Franco, Reyes \& Cuadrado, 2017), mientras que otros estudios indican que no se relacionan (Pacheco, 2019), o que el LF no predice ninguna de las dimensiones de SL; bajo ese panorama, contribuir con investigaciones y dar evidencia científica ayudará a generar posibles estudios sobre ambas variables que permitan determinar la relación entre estas variables, es decir, responder a la interrogante de si existe o no existe relación o predicción entre estas variables. Cabe mencionar que en la realidad peruana es reducida el número de investigaciones que relacionen ambas variables. Según la literatura revisada, existen estudios en su mayoría de carácter correlacional, es decir, aquella que muestra solo relaciones aleatorias, pero no predictivas. Así mismo, se observó que la mayoría de investigaciones en el ámbito educativo emplean muestras específicas de docentes y no toman en cuenta al personal de las diferentes áreas que también forman parte de la institución educativa. 
Por otra parte, la presente investigación se justifica de manera práctica, porque contribuye al reconocimiento de la importancia de las habilidades de liderazgo en los directivos en centros educativos; lo cual repercute en la satisfacción laboral del personal y estudiantes. Por ello, la presente investigación, permitirá a las instituciones educativas mejorar y potenciar el estilo de dirección que contribuya al desarrollo de la gestión educativa. Además, este tipo de investigaciones, aportan beneficios en la gestión del clima organizacional, productividad y ausentismo (Hantula, 2015; Madanchian \& Noordin, 2017; Tohidi \& Jabbari, 2012).

\section{Objetivos}

\section{Objetivo general}

Identificar si los EL predicen las dimensiones de la SL en del personal administrativo de una Institución Educativa Superior de Lima y Arequipa.

\section{Objetivos específicos:}

- Identificar si el estilo de LTF es predictor de las dimensiones de SL en el personal administrativo de un Instituto de Educación Superior de Lima y Arequipa.

- Identificar si el estilo de LT es predictor de las dimensiones de SL en el personal administrativo de un Instituto de Educación Superior de Lima y Arequipa.

- Identificar si el estilo de liderazgo LF es predictor de las dimensiones de SL en el personal administrativo de un Instituto de Educación Superior de Lima y Arequipa.

Como hipótesis general del trabajo, se espera que los EL predigan la SL del personal administrativo de una Institución Educativa Superior de Lima y Arequipa.

\section{Hipótesis}

\section{Hipótesis general}


Los estilos de liderazgo predicen la SL del personal administrativo de una Institución Educativa Superior de Lima y Arequipa.

\section{Hipótesis especificas}

1. El LTF es predictor positivo de las dimensiones de SL en el personal administrativo de un Instituto de Educación Superior de Lima y Arequipa.

2. El LT es predictor positivo de las dimensiones de SL en el personal administrativo de un Instituto de Educación Superior de Lima y Arequipa.

3. El liderazgo LF es predictor negativo de las dimensiones de SL en el personal administrativo de un Instituto de Educación Superior de Lima y Arequipa. 


\section{Método}

\section{Tipo y diseño de investigación}

La presente investigación tiene un diseño empírico, de estrategia asociativa y de estudio predictivo transversal, según Ato, López y Benavente (2013) porque se pretende explicar la relación funcional entre las variables EL y SL.

\section{Participantes}

La población estuvo conformada por 120 trabajadores de un Instituto de Educación Superior que forma profesionales para el sector minero. La muestra fue seleccionada a través de métodos no probabilístico de tipo conveniencia (Hernandez, Fernandez \& Baptista, 2010), esto debido a que accesibilidad y proximidad de la muestra. Se contó con 84 trabajadores con un rango de edad desde 21 a 71 años $(M=38.1)$, donde 55 fueron varones $(65.5 \%)$ y 29 mujeres (34.5\%); los tipos de contrato existentes en la empresa son: intermitente $(n=39 ; 46.4 \%)$, a plazo fijo $(n=29 ; 34.5 \%)$ e indeterminado $(n=16 ; 19 \%)$. De acuerdo al estado civil los participantes

fueron solteros $(n=44 ; 52.4 \%)$; casados $(n=35 ; 41.6 \%)$; divorciado $(n=5 ; 6 \%)$. La segmentación de acuerdo a las áreas laborales a las que pertenecen es: académico $(n=55 ; 65.5 \%)$, administración $(n=10 ; 11.9 \%)$, comercial $(n=12 ; 14.3 \%)$ y operaciones $(n=7 ; 8.3 \%)$. De acuerdo a la sede, la distribución fue para Lima de $(n=66 ; 78.6 \%)$ y Arequipa de $(n=18 ; 21.4 \%)$.

Entre los criterios de inclusión, se consideró el período de permanencia en la empresa y en el puesto, el cual tuvo que ser mayor a seis meses, y además que el tiempo de reporte al jefe inmediato tuvo que ser mayor a tres meses. Los criterios de exclusión definidos fueron; personal que no estén dentro del rango de edad, personas con periodo de permanencia en la empresa y en 
el puesto, y tiempo de reporte al jefe inmediato menor a 3 meses, y no se consideró al personal que no se encuentre en la planilla de la organización.

\section{Instrumentos}

Para la medición de la variable EL se empleó el Cuestionario de EL CELID forma S. Esta prueba tiene sus cimientos en la prueba de Multifactor Leadership Questionnaire (MLQ) de Bass y Avolio (2004). Fue construido por Castro, Nader, y Casullo (2004), y validado en Perú por Quispe (2020). Tiene como objetivo medir los EL a través de 36 items con cinco opciones de respuesta de tipo Likert desde $1=$ totalmente en desacuerdo, hasta $5=$ totalmente de acuerdo. Los ítems se dividen en estilo de LTF (diecisiete items), LT (once ítems) y LT (seis items). En la investigación de Quispe (2020), se encontró que la validez se obtuvo mediante jueces de expertos y la confiabilidad para el estilo de LTF fue $\alpha=.966$, para el estilo LT fue $\alpha$ $=.690$ y para LF fue $\alpha=.778$.

Para la evaluación de la variable SL se utilizó el Cuestionario de Satisfacción S21/26 (Meliá et al., 1990), que fue validado por Dominguez, Calderón y Arroyo (2016). Este instrumento tiene como objetivo medir el auto reporte de la percepción de la satisfacción en el contexto laboral, posee 20 items con cinco opciones de respuesta de tipo Likert que va desde 1= totalmente en desacuerdo, hasta $5=$ totalmente de acuerdo. Las dimensiones de la variable SL son: SI (cinco ítems), SS (cinco items), SCP (cuatro items) y SP (seis items). En el estudio de Dominguez et al. (2016) reportaron un procedimiento de V de Aiken para identificar la validez de contenido, y para la validez de la estructura interna se realizó un análisis factorial exploratorio. La confiabilidad fue obtenida para la SI $(\alpha=.855)$, SS $(\alpha=.807)$, SCP $(\alpha=.785)$ y SP $(\alpha=.757)$. 


\section{Procedimientos}

La técnica empleada para la recolección de datos fue la encuesta autoadministrada virtual con un tiempo aproximado de resolución de 15 minutos. Para este proceso se solicitó la carta de presentación por parte de la Universidad San Ignacio de Loyola, a fin de garantizar a la Institución Educativa Superior que la información será confidencial y será analizada de manera responsable. Seguidamente se presentó la propuesta de investigación y se solicitó el permiso para la aplicación de la encuesta. Después, se acordó con el Directorio y el área de Recursos Humanos para que puedan difundir la encuesta mediante su canal de comunicación interna. El periodo de la evaluación duró cuatro días hábiles a través de un formulario virtual, dentro de las cuales los participantes antes de iniciar la evaluación dieron su consentimiento informado en el cual por aspectos éticos, se indicaba los objetivos de la investigación, que la participación era voluntaria, anónima y que la información solo será utilizada con fines de la presente investigación y en el caso de ser publicado el estudio no se revelará ningún dato de los participantes y la empresa. Finalmente, una vez concluida la investigación y sustentación se entregará un informe final a la gerencia de la institución con los resultados finales y plan de acción.

\section{Análisis de datos}

El análisis de la base de datos se realizó mediante la confiabilidad por medio del coeficiente Alpha de Crombach $(\alpha)$ donde valores menores a .50 son inaceptables, entre .50 y .60 resulta pobre, entre .60 y .70 son cuestionables, entre .70 y .80 son aceptables, entre .80 y .90 son buenos y de.90 a .99 se consideran excelentes. (George \& Mallery, 2003). Seguidamente se analizó los supuestos de normalidad (asimetría y curtosis) las cuales al arrojar resultados entre +/- 1.5 indican que los datos se aproximan a una distribución normal. También se realizó la 
correlación de Pearson entre las dimensiones de los EL con la SL donde valores tanto positivos y negativos alrededor de $r=.50$ son correlaciones medias, alrededor de $r=.75$ considerables, de $\mathrm{r}=.90$ muy fuerte y correlaciones $=1$ son perfectas (Hernández, Fernández, \& Baptista, 2010). Además, se realizó el análisis de regresión lineal con la finalidad de conocer la variabilidad en las puntuaciones de SL que es descrita por los cambios en las dimensiones de Los EL, para ello se interpretó el coeficiente $\mathrm{R}^{2}$ para comprender el porcentaje de varianza explicada por la variable independiente y se interpretó los coeficientes beta $(\beta)$ donde valores < |.20|, entre 20 y 50 y >.80 calificado como débil, moderada y fuerte respectivamente (Domínguez, 2017 


\section{Resultados}

\section{Análisis descriptivos}

El análisis de confiabilidad para la variable EL fue: LTF $\alpha=.956$, LT $\alpha=.767$ y LF $\alpha=$ .761. Por otro lado, para la variable SL se obtuvo los siguientes resultados: SI $\alpha=.783$, SS $\alpha=$ $.927, \operatorname{SCP} \alpha=.789$ y SP $\alpha=.851$.

\section{Análisis de normalidad}

La tabla 1, muestra los valores de asimetría y curtosis por cada dimensión de las variables, donde se observa que la desviación de los datos con relación a la normalidad univariada no fue muy elevada, ya que la magnitud de la asimetría (g1) y curtosis (g2) en la mayoría de los casos se encuentra dentro de un rango razonable (+/- 1.5; Pérez \& Medrano, 2010).

Tabla 1

Asimetría y curtosis

\begin{tabular}{ccc}
\hline & $g 1$ & $g 2$ \\
\hline LTF & -.554 & .322 \\
LT & .093 & .323 \\
LF & .503 & -.075 \\
SI & -.910 & .721 \\
SCP & -.400 & .028 \\
CP & .239 & -.214
\end{tabular}

Nota: $g 1$ = asimetría $;$ g = curtosis; $L T F=$ liderazgo transformacional; $L T$ = liderazgo transaccional; $L F=$ laissez faire; $S I=$ satisfacción intrínseca; $S C P=$ satisfacción con la calidad de producción, $S P=$ satisfacción con la participación

La tabla 2, muestra los valores de correlación de las dimensiones de EL y la SL. la correlación entre el LTF y la SI y la SCP fueron estadísticamente significativas positivas de magnitud moderada, mientras que, con la SS y SP fueron positivas de magnitud alta. 
Por otro lado, las correlaciones entre el liderazgo LF con la SI y la SS fueron estadísticamente significativas negativas de magnitud baja. Finalmente, el estilo de LT solo tiene correlación estadísticamente significativa positiva de magnitud moderada con la SP.

Tabla 2

Correlaciones entre las variables EL y SL

\begin{tabular}{ccccc}
\hline & & LTF & LT & LF \\
\hline \multirow{2}{*}{ SI } & $\mathrm{r}$ & .447 & .036 & -.223 \\
& $\mathrm{p}$ & .001 & .747 & .042 \\
$\mathrm{~S} S$ & $\mathrm{r}$ & .770 & .133 & -.373 \\
& $\mathrm{p}$ & .001 & .227 & .001 \\
$\mathrm{SCP}$ & $\mathrm{r}$ & .536 & .117 & -.148 \\
& $\mathrm{p}$ & .001 & .288 & .180 \\
$\mathrm{SP}$ & $\mathrm{r}$ & .789 & .633 & -.106 \\
& $\mathrm{p}$ & .001 & .001 & .335
\end{tabular}

Nota: $r=$ correlación de Pearson; $p$ = significancia estadística; SI=satisfacción intrínseca; $S S=$ satisfacción con la supervisión; $S C P=$ satisfacción con la calidad de producción, $S P=$ satisfacción con la participación; $L T F=$ liderazgo transformacional; $L T=$ liderazgo transaccional; $L F=$ laissez faire

\section{Análisis de regresión}

En la tabla 3, se observa que el LTF y LF explican el 20.1\% de la variación de la SI y que solo el LTF es un predictor moderado positivo estadísticamente significativo. Estos resultados son evidencias de respaldo para la hipótesis específica 1 y en contra para la hipótesis específica 3 .

Tabla 3

Análisis de regresión para SI

\begin{tabular}{cccc}
\hline & B & $\beta$ & $p$ \\
\hline LTF & 6.87 & .43 & .001 \\
LF & -.029 & -.003 & .735 \\
\hline
\end{tabular}

Nota: $B=$ coeficiente no estandarizado; $\beta=$ coeficiente beta $;$ LTF = liderazgo transformacional $;$ LF = laissez faire 
En la tabla 4, se observa que el LTF y LF explican el 59.5\% de la variación de la SS y que solo el LTF es un predictor fuerte positivo estadísticamente significativo de SS. Estos resultados son evidencias de respaldo para la hipótesis específica 1 y en contra para la hipótesis específica 3.

Tabla 4

Análisis de regresión para SS

\begin{tabular}{cccc}
\hline & B & $\beta$ & $p$ \\
\hline LTF & .276 & .748 & .001 \\
LF & -.049 & -.05 & .520
\end{tabular}

Nota: $\mathrm{B}=$ coeficiente no estandarizado; $\beta=$ coeficiente beta; $\mathrm{LTF}=$ liderazgo transformacional; $\mathrm{LF}=$ laissez faire

En la tabla 5, se observa que el LTF explica el $28.7 \%$ de la variación de la SCP y que es un predictor moderado estadísticamente significativo. Estos resultados son evidencias de respaldo para la hipótesis específica 1.

Tabla 5

Análisis de regresión para SCP

\begin{tabular}{cccc} 
& B & $\beta$ & $p$ \\
\hline LTF & .122 & .536 & .001
\end{tabular}

Nota: $B=$ coeficiente no estandarizado; $\beta=$ coeficiente beta; $L T F=$ liderazgo transformacional

En la tabla 6 se observa que el LTF y LT explican el 81.1\% de la variación de la SP, en este caso, el LTF es un predictor fuerte estadísticamente significativo y el LT es un predictor moderado. Estos resultados son evidencias de respaldo para la hipótesis específica 1 e hipótesis específica 2.

Tabla 6 


\section{Análisis de regresión para SP}

\begin{tabular}{cccc}
\hline & B & $\beta$ & $\mathrm{p}$ \\
\hline LTF & 0.182 & .666 & .001 \\
LT & 0.243 & .452 & .001
\end{tabular}

Nota: $B=$ coeficiente no estandarizado; $\beta=$ coeficiente beta,$L T F=$ liderazgo transformacional $; L T=$ liderazgo transaccional 


\section{Discusión}

En el contexto actual las variables EL y SL repercuten en el éxito de las organizaciones y el desarrollo de sus trabajadores porque se relacionan con otras variables como el compromiso organizacional, ausentismo, desempeño laboral, clima organizacional, efectividad percibida, motivación, creatividad y flexibilidad, productividad y adaptación al cambio (Álaval et al., 2016; Bitsch \& Hogberg, 2005; Hermosilla et al., 2016; Sanin \& Salanova, 2013).De esto se puede derivar que ambas variables tienen fuertes implicancias para la persona y la organización, por ello, se planteó como objetivo de investigación determinar si los EL predicen la SL en el personal administrativo de un Instituto Superior de Lima y Arequipa.

Los resultados evidenciaron que el estilo de LTF predice positivamente de manera moderada y fuerte las dimensiones de la SL. Mientras que el estilo de LT predice solo la SP, de manera moderada, significativa y positiva. Finalmente, el estilo de liderazgo LF no predice ninguna de las dimensiones de la SL.

En cuanto al estilo de LTF, los resultados apoyan a la hipótesis específica 1, es decir que este estilo predice de forma positiva y significativa las dimensiones de la SL. Estos resultados se pueden explicar porque los líderes con este estilo emplean su energía y entusiasmo para motivar, reconocer logros, bridan mayor autonomía y libertad para afrontar con éxito situaciones conflictivas, tolerancia y oportunidades de desarrollo; también otorgan nuevos retos y aspiraciones de crecimiento a los trabajadores, que son características de la SI, (Bass, 1985, 1990; Bass \& Avolio, 1994, 2006; Burns, 1978; Meliá et al., 1990; Vargas \& Mayne, 2019). Esto puede generar un ambiente de confianza respeto e igualdad entre los integrantes del equipo de trabajo y el líder, mediante una comunicación organizacional horizontal, que es una de las 
características de la SS (Flores \& Salazar, 2019; Meliá et al., 1990). Las características del LTF también generan que los trabajadores perciban que la organización brinda los recursos y medio necesarios para realizar sus funciones en el puesto laboral, y de esta forma el trabajador podrá cumplir con metas y objetivos deseados, lo cual permitirá obtener el reconocimiento correspondiente, siendo estas, características de la SCP (Kellogg et al., 1997; Meliá et al., 1990). Finalmente, el LTF brinda formación por parte de la empresa y autonomía en la toma de decisiones, lo que puede originar que un trabajador tenga libertad de emitir juicios valorativos sobre aspectos de la organización orientados al cumplimiento individual y colectivo de las metas, las cuales con características de la dimensión SP (Bass \& Peiró, 1989; López \& Magallanes, 2017; Meliá et al., 1990).

Por otro lado, estos resultados encontrados coinciden con los estudios de Franco, Reyes y Cuadrado (2017), Redolfo (2017), Chacón (2016), Saleem (2015), Paucar (2013), Samson (2016), Ramandeep, Vaishali y Neetu (2016), que muestran correlaciones significativas positivas entre las variables, lo que indica que las personas que perciben a sus líderes inmediatos con este estilo presentan mayores niveles de SL. Sin embargo, estos resultados contradicen lo hallado por Pacheco (2019) quien encontró que, no existe correlación significativa entre LTF y SL. Esta diferencia en los resultados puede deberse a que en el estudio de Pacheco (2019), el objetivo del instrumento evalúa un solo estilo de liderazgo (LTF), lo cual según el reporte del investigador no existe un único EL dentro de la organización, desencadenando que los trabajadores evalúen el estilo de liderazgo deseado mas no las características reales de su líder. Por el contrario, el instrumento de la presente investigación evalúa diferentes estilos de liderazgo, lo que permite al evaluado identificar en su líder, las características de los estilos existentes. 
Además, otro estudio de Gallegos y Miranda (2019) encontró que LTF predice todas las dimensiones de SL de manera positiva y significativa, a excepción de la SP, resultado que se diferencia parcialmente con lo encontrado en la presente investigación, que muestra que el LTF predice todas las dimensiones de la SL. Esto puede deberse a ciertas diferencias sociodemográficas de los sujetos evaluados en ambos estudios, es decir, en el estudio de Gallegos y Miranda (2019) los sujetos pertenecen a diferentes empresas, lo cual podría reflejar diferentes realidades, por el contrario, en nuestro estudio los sujetos pertenecen a una única empresa que si bien no cuenta con programas de capacitación y desarrollo, los trabajadores tienen autonomía para la toma de decisiones y participación en las ideas de mejora para organización.

En cuanto al estilo de LT, los resultados respaldan parcialmente la hipótesis específica 2, porque este estilo solo predijo de manera positiva y significativa la dimensión de SP. Esto puede deberse a que este estilo está enfocado en el proceso de intercambio de recompensas entre el líder y el seguidor. Por ello, aquellos trabajadores que tienen la autonomía para participar en la toma de decisiones se sienten recompensados por su trabajo y por ende presentan mayores niveles de satisfacción (Bass, 1999, 2007; López \& Magallanes, 2017; Quispe, 2020; Varela, 2010). Según informe interno del área de RRHH de la muestra de la presente investigación, por lo general los líderes instauran dentro de la organización ciertos patrones de conducta y fijan ciertas reglas y metodologías implícitas de trabajo que los integrantes del grupo deben cumplir, y si estos las cumplen reciben atención, consideración en la toma de decisiones y capacitación por parte del líder; estas características del líder permiten la SP. Por el contrario, si no las cumplen, no reciben atención y autonomía generando insatisfacción con la participación en el evaluado. 
Esto coincide con algunas investigaciones que confirman que el LT predice de manera significativa y positiva las dimensiones de la SL entre ellas la dimensión de SP (Franco, Reyes \& Cuadrado, 2017; Gallegos \& Miranda, 2019; Oliva \& Molina, 2016; Ramandeep, Vaishali \& Neetu, 2016; Salem, 2015; Samsom, 2016). Esto se explica porque el líder transaccional adopta un rol de trato justo e igualitario con sus seguidores, es decir, motiva a partir de recompensas u otros refuerzos como la autonomía para tomar decisiones, brindar sugerencias, participar en programas de capacitación, pago de horas extras y bonos que reconocen el desempeño de sus seguidores.

No obstante, estos resultados se contradicen a los encontrados por Paucar (2013) que el LT presenta una relación inversa con la SL, eso quiere decir que, a mayor presencia de este estilo, menores niveles de SL. Las diferencias entre la presente investigación y la de Paucar (2013) se puede deber a que el autor empleó una muestra menor $(\mathrm{n}=36)$ que a su vez pertenecían a diferentes instituciones del sector público, por ende, se deben regir a las normas planteadas por la UGEL y la DREP, limitando la autonomía en la toma de decisiones. Sin embargo, en el presente estudio la muestra empleada fue mayor $(\mathrm{n}=84)$ y se enfocó en una única institución del sector privado, permitiendo mayor autonomía en los procedimientos y normas.

Por otro lado, Ramandeep, Vaishali y Neetu (2016), halló que el LT tiene un efecto negativo en la SL. Estos resultados resultan contradictorios a los de la presenta investigación, porque el autor incluyo un tamaño de muestra de género femenino mayor $(n=421)$ de diferentes empresas pertenecientes a sectores público y privado de rubros como: salud, banca, educación y aseguradoras. Cabe recordar que la presente investigación incluyó únicamente una muestra del sector educativo y con un tamaño menor. 
Por último, los resultados no respaldan la hipótesis específica 3, ya que el estilo LF no es predictor de ninguna de las dimensiones de la SL, porque este estilo se caracteriza por la poca o nula participación de los líderes en la toma de decisiones, supervisión de la tarea, motivación de procesos, justicia procedimental, entre otros, desencadenando que los subordinados sean quienes asumen el protagonismo y responsabilidad (Bass \& Riggio, 2006; Rodríguez, Gil \& Moreno, 2012). Esto se puede explicar también, por la especificidad de la muestra, ya que la institución evaluada pertenece al sector minero y por lo general se observa perfiles de comportamiento autoritario y participativo en los ingenieros e instructores. Además, los líderes buscan el cumplimiento de los objetivos a través de las normativas según el sector al que pertenecen, lo cual se contradice con las características de un líder con un estilo LF. Estos resultados se avalan con los estudios realizados por Gallegos y Miranda (2019).

Sin embargo, existen autores que indican que el estilo LF correlaciona de manera negativa con la SL, (Franco, Reyes \& Cuadrado, 2017; Oliva \& Molina, 2016; Paucar, 2013; Ramandeep, Vaishali \& Neetu, 2016). Por su parte, en el estudio de Franco, Reyes y Cuadrado (2017), Oliva y Molina (2016) las diferencias que podría explicar estos resultados son el tamaño de la muestra, el tipo de puesto (administrativos y operativos), especialidades y los participantes pertenecen a diferentes empresas y centros; mientras que la presente investigación se enfoca en un área administrativa, con un tamaño de muestra menor y la institución pertenece al rubro educativo. Así mismo, se puede explicar los resultados del estudio de Paucar (2013) porque presentó una muestra pequeña $(\mathrm{n}=36)$ y de diferentes instituciones del sector educativo. Del mismo modo, en el estudio de Ramandeep, Vaishali y Neetu (2016) la muestra empleada fue mayor $(n=421)$ y se enfocó en el personal femenino con cargos superiores en diferentes 
empresas y rubros de la India; lo cual difiere con las características sociodemográficas de la presente investigación.

El presente estudio permitirá a la institución educativa conocer la proporción de los EL en los directivos y su influencia en el grado de SL. Esta información permitirá al directorio tomar decisiones sobre qué estilo de liderazgo es el que más se ajusta para el logro de los objetivos institucionales y además permitirá instaurar el LTF como perfil de liderazgo de los directivos, porque existe suficiente evidencia teórica y práctica sobre los efectos positivos de este estilo en la SL, desempeño laboral, productividad laboral, comunicación organizacional, clima organizacional y reducción de la rotación de personal (Álaval et al., 2016; Bitsch \& Hogberg, 2005; Hermosilla et al., 2016; Sanin \& Salanova, 2013).

A nivel teórico, el estudio aporta información que fundamenta que el estilo de LTF predice de manera significativa y positiva todas las dimensiones de la SL, lo cual es evidencia a favor de la interacción de estas variables al igual que los estudios de Paucar (2013), Gallegos y Miranda (2019) y Franco, Reyes y Cuadrado (2017). Sin embargo, el estilo LT solo predice la SP y finalmente confirma que no existe evidencia significativa que avale que el estilo de LF predice la SL. Estos datos necesitan de mayores estudios en otras muestras para corroborar si esta baja predicción del LT y LF se replica en otros contextos, lo cual puede ser evidencia de que el LTF es el estilo más importante y de mayor impacto en los trabajadores y las organizaciones.

Esta investigación tuvo limitaciones, como: la disponibilidad de la muestra, debido a que la recolección de datos se hizo de manera virtual y voluntaria, por lo que ciertos participantes no pudieron completar la encuesta. En ese sentido la muestra deseada se vio reducida en un $30 \%$. Por parte de los trabajadores se puede considerar como limitación el uso del cuestionario 
virtual enviado por el Área de Recursos Humanos porque esto podría haber alterado las respuestas de los participantes por la deseabilidad social y presión por reprimendas por su jefe inmediato. Adicionalmente, la cantidad de los sujetos evaluados no fue equitativa en las sedes, ya que la sede de Lima cuenta con una mayor cantidad. Así mismo, la cantidad de participantes fue diversa siendo el área académica una de las más representativas. Además, otra de las limitaciones fue la amplitud de la encuesta virtual y el periodo de evaluación que fue interferida por temas administrativos de la institución y se otorgó cuatro días de evaluación.

\section{Conclusiones y recomendaciones}

Por último, se considera el escaso número de investigaciones previas con las características del presente estudio, porque se empleó una muestra particular.

- En relación con la primera hipótesis, ésta fue comprobada en su totalidad, porque el estilo de LTF predice de manera positiva y significativa todas las dimensiones de la SL.

- En relación a la segunda hipótesis, ésta fue comprobada de manera parcial, porque el estilo de LT predice de manera positiva y significativa únicamente la dimensión SP.

- En relación a la tercera hipótesis, el liderazgo LF no predice ninguna de las dimensiones de la SL.

- Se sugiere desarrollar investigaciones sobre liderazgo en docentes y satisfacción con la enseñanza percibida por estudiantes del mismo sector. Esto debido a que existen estudios en las que demuestran que el estilo de liderazgo participativo y persuasivo afecta 
positivamente en la felicidad de los estudiantes; mientras que un estilo directivo y delegativo produce una felicidad neutra (Correa \& Cuevas, 2017).

- Por otra parte, se sugiere a futuras investigaciones emplear una mayor cantidad de muestra para garantizar la validez interna y externa de la investigación. Así mismo, poder considerar comparaciones en muestras variadas como; instituciones privadas y públicas del sector, áreas de trabajo, puesto de trabajo, tiempo de servicio (Ramandeep, Vaishali \& Neetu, 2016; Chiang, Gómez \& Salazar, 2014; Pacheco, 2019).

- También se recomienda añadir nuevas variables como la personalidad, generaciones sociales, compromiso organizacional, desempeño organizacional, clima organizacional que puedan ayudar a entender la relación entre El y SL. Tales como los estudios de Chiang y Ojeda (2011); Montoya, et al. (2017); Pedraza (2020) explican que aquellos trabajadores que se encuentran satisfechos con la labor que realizan, las relaciones con sus superiores y pares, satisfacción con el reconocimiento muestran mayores niveles de productividad y desempeño. 


\section{Referencias}

Almirón, V., Tikhomirova, A., Trejo Toriz, A.C., \& García, J.M. (2015). Liderazgo transaccional vs liderazgo transformacional. Reidocrea, 4, 24-27. Recuperado de: http://hdl.handle.net/10481/34629

Amarillo, J. D., \& Mosquera, M. C. (2012). Atracción y retención de empleados y su relación con la satisfacción laboral (Tesis de licenciatura). Recuperado de: https://intellectum.unisabana.edu.co/handle/10818/1670

Anaya, D., \& López, E. (2015). Satisfacción laboral del profesorado de educación secundaria. Revista De Investigación Educativa, 33(2), 435-452. Recuperado de: https://doi.org/10.6018/rie.33.2.202841

Ato, M., López, J. J., \& Benavente, A. (2013). Un sistema de clasificación de los diseños de investigación en psicología. Anales de Psicología, 29(3), 1038-1059.

Avolio, B. J. (2007). Promoting more integrative strategies for leadership theorybuilding. American Psychologist, 62(1), 25-33. Recuperado de: https://doi.org/10.1037/0003-066X.62.1.25

Avolio, B., Walumbwa, F., \& Weber, T J. (2009). Leadership: Current Theories, Research, and Future Directions. Management Department Faculty Publications. 37. Recuperado de: https://digitalcommons.unl.edu/managementfacpub/37

Babalola, S. S. (2016). The effect of leadership style, job satisfaction and employee-supervisor relationship on job performance and organizational commitment. Journal of Applied Business Research (JABR), 32(3), 935-946.

Bass, B., \& Avolio, B. (2006a). Manual for the multifactor leadership questionnaire. Estados Unidos: Consulting Psychologist Press.

Bass, B. M. (1990). From Transactional to Transformational Leadership: Learning to Share the Vision. Organizational Dynamics, 18, 19-32. Recuperado de: http://dx.doi.org/10.1016/0090-2616(9090061-S

Bass, B. M., \& Avolio, B. J. (1995). Multifactor Leadership Questionnaire (MLQ). Full Range Leardership. Recuperado de: https://doi.org/10.1037/t03624-000 
Bass, B. M., \& Riggio, R. E. (2006). Transformational Leadership (2nd ed.). Recuperado de: https://play.google.com/store/books/details?id=2WsJSw6wa6cC

Berdecía, Z. I., \& González, J. R., Carrasquillo (2013). Estilos de Liderazgo para el Éxito Organizacional: Estudio de Casos Múltiple en Empresas Puertorriqueñas. Revista de Estudios Avanzados de Liderazgo, 1(2), 21-32. Recuperado de: https://www.regent.edu/acad/global/publications/real/vol1no2/cruz.pdf

Barajas, L., Betancur, L., Lopez, N., \& Rodríguez, J. (2020). Liderazgo en tiempos de pandemia. Universidad cooperativa de Colombia. Recuperado de: https://repository.ucc.edu.co/bitstream/20.500.12494/19790/1/2020_liderazgo_tiempos_p andemia.pdf

Carranza, M. L. (2019). Los estilos de liderazgo y los estilos de pensamiento en los directivos de Instituciones Educativas. Big Bang Faustiniano Revistas, 8(4), 37-41. Recuperado de: http://revistas.unjfsc.edu.pe/index.php/BIGBANG/article/view/561/542

Castillo, E. F., Medina, M. A., Bernardo, J. V., Reyes, C. E., \& Ayala, C. I. (2019). Liderazgo y clima organizacional en trabajadores de establecimientos de salud de una microred de Perú. Revista cubana de salud pública, 45(2), 1-13. Recuperado de: http://repositorio.ucv.edu.pe/handle/20.500.12692/37332

Carhuachin, E. (2019). La satisfacción laboral como predictora del desempeño laboral percibido en una Pyme de lima. (Tesis de licenciatura), Universidad San Ignacio de Loyola. Recuperado de: http://repositorio.usil.edu.pe/bitstream/USIL/9725/1/2019_CarhuachinMaximiliano.pdf

Cernas, z, D. A., Mercado, P., \& Davis, M. A. (2018). Perspectiva futura de tiempo, satisfacción laboral y compromiso organizacional: el efecto mediador de la autoeficacia, la esperanza y la vitalidad. Journal of Work and Organizational Psychology, 34(1), 1-9. Recuperado de: https://doi.org/10.5093/jwop2018a1

Chiang, M., Gómez, N., \& Hidalgo, J. (2017). Job satisfaction of Chilean workers. A model of structural equations. Cuadernos de Administración, 33(57), 48-60. Recuperado de: http://www.scielo.org.co/scielo.php?pid=S012046452017000100048\&script=sci_arttext\& tlng=en 
Chiang, M., Ojeda, J. (2011). Estudio de la relación entre satisfacción laboral y el desempeño de los trabajadores de las ferias libres. Contaduría y administración. 58. 39-60. Doi: 10.1016/S0186-1042(13)71209-9.

Chiavenato, I. (2002). Gestión del talento humano. México D.F: Mc Graw Hill.

Comisión Económica para América Latina y el Caribe (2020). Los efectos económicos y sociales del COVID-19 en América Latina y el Caribe. Recuperado de: https://www.cepal.org/sites/default/files/presentation/files/200605_final_presentacion_par lamericasv_alicia_barcena.pdf

Correa, A., \& Cuevas, R. (2017). Liderazgo docente y percepción de felicidad en estudiantes de educación superior. Revista especializada en ciencias de la salud 20(01), 20-26. Recuperado de: https://www.medigraphic.com/pdfs/vertientes/vre-2017/vre171c.pdf

Da Costa, S., Oriol, X., Etchebehere, G., \& Hermosilla, D. (2019). Factores favorables y constrictores de la creatividad e innovación en organizaciones socioeducativas de España y Sud-América. Veritas \& Research, 1(2), 71-83.

Decreto Supremo N 010-2020-TR. (2020) Decreto Supremo que desarrolla disposiciones para el Sector Privado, sobre el trabajo remoto previsto en el Decreto de Urgencia $N^{\circ} 026-2020$, Decreto de Urgencia que establece medidas excepcionales y temporales para prevenir la propagación del COVID - $19 . \quad$ Recuperado de: https://www.gob.pe/institucion/mtpe/normas-legales/462424-010-2020-tr

Dominguez, S. (2017). Magnitud del efecto, una guía rápida. Educación Médica, 19(4), 191 256. Recuperado de: https://www.elsevier.es/es-revista-educacion-medica-71-articulomagnitud-del-efecto-una-guia-S1575181317301390

Dominguez, S., Calderon, G., \& Arroyo, F. (2016). Cuestionario de Satisfacción S21/26: Análisis psicométrico preliminar en trabajadores administrativos. Revista de Investigación - UCSP, 7, $51-67$.

Escandon, D. M., \& Hurtado, A. (2016). Influencia de los estilos de liderazgo en el desempeño de las empresas exportadoras colombianas. Estudios gerenciales, 32(139), 137-145. Recuperado de: https://www.sciencedirect.com/science/article/pii/S0123592316300183

Franco A. M., Reyes M. L., \& Cuadrado, S. L. (2017). Incidencia de los Estilos de Liderazgo en la Satisfacción de los Colaboradores en Empresas de Servicios del Ecuador. PODIUM, 41 - 64. Recuperado de: https://revistas.uees.edu.ec/index.php/Podium/article/view/73 
Frias-Navarro, D. (2020). Apuntes de consistencia interna de las puntuaciones de un instrumento de medida. Universidad de Valencia. España. Disponible en: https://www.uv.es/friasnav/AlfaCronbach.pdf

Gamero, H. (2013). La Satisfacción Laboral como Dimensión de la Felicidad. Ciencia \& trabajo, 15(47), 94-102. https://dx.doi.org/10.4067/S0718-24492013000200010

García, R., Fernández, J., Gonzales, E., \& Polo, S. (2017). Asociación del clima organizacional y la satisfacción laboral en empresas de servicios. Revista Internacional Administración \& Finanzas, 10 (1) 37-48. Recuperado de: https://ssrn.com/abstract=2916923

González, F., López, T., \& Sánchez, S. (2013). Satisfacción laboral y compromiso organizativo en los recursos humanos de la hostelería de Córdoba (España): influencia de la tipología contractual y la jornada de trabajo. Intangible capital, 10(1), 189-211.

Hantula, D. A. (2015). Job satisfaction: The management tool and leadership responsibility. Journal of Organizational Behavior Management, 35(1-2), 81-94. doi: 10.1080/01608061.2015.1031430

Instituto Nacional de Estadística e Informática (2020) La población ocupada del país alcanzó 10 millones 272 mil 400 personas en el II trimestre del 2020. Recuperado de:https://www.inei.gob.pe/media/MenuRecursivo/noticias/nota-de-prensa-no-117-2020inei.pdf

Imirón Arévalo, V., Tikhomirova, A., Trejo Toriz, A.C., \& García-Ramírez, J.M. (2015) Liderazgo transaccional vs liderazgo transformacional. Reidocrea, 4, 24-27 Recuperado de: http://hdl.handle.net/10481/34629

Locke, E. A. (1969). What is Job Satisfaction? Organizational Behavior and Human Performance. ScienceDirect, 4, 309-336. doi: 10.1016/0030-5073(69)90013-0

Lupano, M., \& Castro, A. (2008). Estudios sobre el liderazgo: Teorías y evaluación. Psicodebate 6: Psicología, Cultura y Sociedad, 8, 107-122. Recuperado de: http://www.palermo.edu/cienciassociales/publicaciones/pdf/Psico6/6Psico\%2008.pdf

Medina, A. M., Salazar, M. L., \& González, S. L. (2017). Incidencia de los EL en la satisfacción de los colaboradores en empresas de servicios del Ecuador. Podium, 41-64. Recuperado de: https://revistas.uees.edu.ec/index.php/Podium/article/view/73

Montoya, P., Beiio-Escamilla, N., Bermúdez, N., Burgos, F., Fuentealba, M., \& Padilla, A. (2017). Satisfacción laboral y su Relación con el Clima Organizacional en Funcionarios de 
una Universidad Estatal Chilena. Ciencia \& trabajo, 19(58), 7-13. Recuperado de: https://dx.doi.org/10.4067/S0718-24492017000100007

Oliva, M., \& Molina. S. (2018). Estilos de liderazgo y satisfacción laboral en trabajadores de establecimientos de atención primaria en salud de la comuna de Chillán. Revista Académica \& Negocios, 2(1), 51-68.

Oliva, M., \& Molina, S. (2016). Estilos de liderazgo y satisfacción laboral en trabajadores de establecimientos de atención primaria en salud de la comuna de Chillán. Revista Academia \& Negocios, 2(01), 51-68. Recuperado de: https://ssrn.com/abstract=2804644

Paucar, L. (2013). Estilos de liderazgo y satisfacción laboral en las instituciones educativas secundarias emblemáticas de la ciudad de Puno. Revista de Investigación Carrera Profesional Obstetricia, 1(2), 20-24. doi: http://dx.doi.org/10.35306/ricpo.v1i2.415

Pedraza, N. A. (2020). El clima y la satisfacción laboral del capital humano: factores diferenciados en organizaciones públicas y privadas. Revista Innovar, 30(76), 9-24. Doi: https://doi.org/10.15446/innovar.v30n76.85191

Pérez, E. R., \& Medrano, L. A. (2010). Análisis Factorial Exploratorio: Bases Conceptuales y Metodológicas. Revista Argentina de Ciencias del Comportamiento, 2(1), 58-66. Recuperado de: https://dialnet.unirioja.es/servlet/articulo?codigo=3161108

Perilla, L. E., \& Gómez, V. (2017). Relación del estilo de LTF con la salud y el bienestar del empleado: el rol mediador de la confianza en el líder. Revista de Psicología Del Trabajo y de Las Organizaciones, 33(2), 95-108. doi: http://dx.doi.org/10.1016/j.rpto.2017.02.005

Quispe, P., \& Cruz, I. C. (2020). EL predominante de los directores de las instituciones educativas estatales red 4 callao. (Tesis de maestría), Universidad San Ignacio de Loyola. Recuperado de: http://repositorio.usil.edu.pe/handle/USIL/9778

Rodríguez, A., Retamal, M., Lizana, J., \& Cornejo, F. (2011). Clima y satisfacción laboral como predictores del desempeño en una organización estatal chilena. Revista Salud y Sociedad, 2(2), 219-234. Recuperado de: https://dialnet.unirioja.es/servlet/articulo?codigo=3899629 Saleem, H. (2018). Los estilos de liderazgo y su efecto en la satisfacción laboral. Procedia Social and Behavioral Science,. 175, 563-569. doi: https://doi.org/10.1016/j.sbspro.2015.01.403 
Sanín, J. A., \& Salanova, M. (2014). Satisfacción laboral: el camino entre el crecimiento psicológico y el desempeño laboral en empresas colombianas industriales y de servicios. Universitas Psychologica, 13(1), 1657-9267. Recuperado de: https://www.redalyc.org/articulo.oa?id=647/64730432009

Tohidi, H., \& Jabbari, M. M. (2012). Organizational culture and leadership. Procedia, Social and Behavioral Sciences, 31, 856-860.

Velásquez, L (2006). Habilidades Directivas y Técnicas de Liderazgo. España: Editorial Ideas Propias. 


\section{Anexo}

\section{Anexo 1:}

\section{Formulario de Consentimiento informado:}

La investigación titulada "Estilos de liderazgo del equipo directivo y satisfacción laboral de personal administrativo en un instituto de formación minera." es conducida por los Bachilleres de psicología Fabiana Trujillo Vera y Virginia Rosales Andagua, perteneciente a la Universidad San Ignacio de Loyola.

Este estudio se realizará en personal administrativo con la finalidad de identificar cómo influyen los estilos de liderazgo que asumen los directivos, en la satisfacción laboral de los mismos. Por ello, su participación es primordial en este proceso y estaremos agradecidos si accede.

El tiempo estimado de la aplicación será 15 minutos. La encuesta es anónima se indagarán sobre los datos generales, satisfacción laboral y estilos de liderazgo. Toda la información recopilada se utilizará para fines del estudio y se tratará con absoluta confidencialidad

Si desea más información, puede enviar un mensaje a virginia.rosales@usilpe. fabiana.trujillo@usil,pe

Se agradece nuevamente por su participación y para iniciar la encuesta darle click en el siguiente botón.

\begin{tabular}{|c|c|c|c|}
\hline 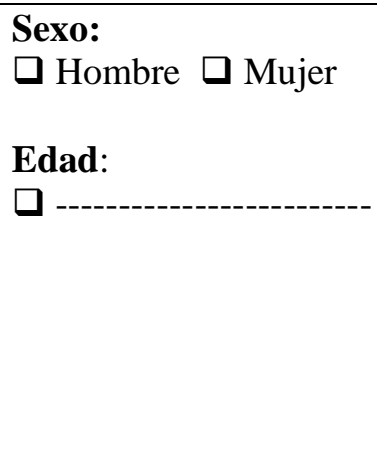 & $\begin{array}{l}\text { Estado Civil: } \\
\square \text { Soltero(a) } \\
\square \text { Casado (a) } \\
\square \text { Conviviente } \\
\square \text { Divorciado (a) } \\
\square \text { Viudo (a) }\end{array}$ & $\begin{array}{l}\text { Grado de instrucción: } \\
\square \text { Técnico Incompleto } \\
\square \text { Técnico en curso } \\
\square \text { Técnico completo } \\
\square \quad \text { Universitario } \\
\text { Incompleto } \\
\square \text { Universitario en } \\
\text { curso Universitario } \\
\square \quad \text { completo }\end{array}$ & $\begin{array}{l}\text { Carrera: } \\
\square \text { - } \\
\text { Cargo: } \\
\square \text {---------. } \\
\text { Sede de labor: } \\
\square \text { Lima } \\
\square \text { Arequipa }\end{array}$ \\
\hline $\begin{array}{l}\text { Área a la que } \\
\text { pertenece: } \\
\square \text { Académico } \\
\square \text { Administración } \\
\square \text { Comercial } \\
\square \text { Operaciones. } \\
\text { Tipo de contrato: } \\
\square \text { Intermitente } \\
\square \text { Plazo fijo } \\
\square \text { Indeterminado }\end{array}$ & $\begin{array}{l}\text { Periodo de permanencia } \\
\text { en el cargo: } \\
\square 3 \text { meses } \\
\square 6 \text { meses } \\
\square 12 \text { meses } \\
\square \text { Más de } 12 \text { meses } \\
\text { Periodo de permanencia } \\
\text { en la sede: } \\
\square 3 \text { meses } \\
\square 6 \text { meses } \\
\square 12 \text { meses } \\
\square \text { Más de } 12 \text { meses }\end{array}$ & $\begin{array}{l}\text { Periodo } \\
\text { permanencia con el } \\
\text { jefe inmediato: } \\
\square 1 \text { mes } \\
\square 3 \text { meses } \\
\square 6 \text { meses } \\
\square \text { Más de } 6 \text { meses } \\
\text { Cargo del jefe } \\
\text { inmediato: } \\
\square \text { - }\end{array}$ & \\
\hline
\end{tabular}


Anexo 2:

\section{Cuestionario de Estilos de Liderazgo (CELID - S)}

(Castro Solano, Nader y Casullo, 2004)

Instrucciones: A continuación, hay una serie de afirmaciones acercar del liderazgo y del acto de liderar. Por favor, indique cuánto se ajusta cada una de ellas al estilo de liderar que usted percibe en su superior. Use la siguiente escala de puntuación y marque con " $\mathrm{X}$ " la alternativa seleccionada. La escala de calificación es la siguiente:

$\mathbf{5}=$ totalmente de acuerdo con la información

$4=$ de acuerdo

$\mathbf{3}=$ ni de acuerdo, ni en desacuerdo

$\mathbf{2}=$ en desacuerdo

$\mathbf{1}=$ totalmente desacuerdo en la afirmación

\begin{tabular}{|c|c|c|c|c|c|c|}
\hline 1 & Su presencia tiene poco efecto en nuestro rendimiento laboral. & 1 & 2 & 3 & 4 & 5 \\
\hline 2 & Evitar cambiar lo que hagamos mientras las cosas salgan bien. & 1 & 2 & 3 & 4 & 5 \\
\hline 3 & Nos sentimos orgullosos de trabajar con él. & 1 & 2 & 3 & 4 & 5 \\
\hline 4 & $\begin{array}{l}\text { Pone especial énfasis en la resolución cuidadosa de los } \\
\text { problemas antes de actuar. }\end{array}$ & 1 & 2 & 3 & 4 & 5 \\
\hline 5 & Evita involucrarse en nuestro trabajo. & 1 & 2 & 3 & 4 & 5 \\
\hline 6 & Evitar decirnos donde se encuentra en algunas ocasiones. & 1 & 2 & 3 & 4 & 5 \\
\hline 7 & $\begin{array}{l}\text { Demuestra que cree firmemente en el dicho "si funciona", no lo } \\
\text { arregles" }\end{array}$ & 1 & 2 & 3 & 4 & 5 \\
\hline 8 & lo que queremos a cambio de recibir su apoyo. & 1 & 2 & 3 & 4 & 5 \\
\hline 9 & Evita intervenir, excepto cuando no se consiguen los objetivos. & 1 & 2 & 3 & 4 & 5 \\
\hline 10 & $\begin{array}{l}\text { Se asegura que exista un fuerte acuerdo entre lo que se espera } \\
\text { que hagamos y lo que puede obtener por nuestro propio esfuerzo. }\end{array}$ & 1 & 2 & 3 & 4 & 5 \\
\hline 11 & $\begin{array}{l}\text { Siempre que sea necesario podemos negociar con él, lo que } \\
\text { obtendremos a cambio de nuestro trabajo. }\end{array}$ & 1 & 2 & 3 & 4 & 5 \\
\hline 12 & $\begin{array}{l}\text { Nos hace saber que podemos lograr lo que queremos si } \\
\text { trabajamos conforme a lo pactado con él. }\end{array}$ & 1 & 2 & 3 & 4 & 5 \\
\hline 13 & Se preocupa de formar a aquellos que lo necesitan. & 1 & 2 & 3 & 4 & 5 \\
\hline 14 & $\begin{array}{l}\text { Centra su atención en los casos en los que no se consigue } \\
\text { alcanzar las metas esperadas. }\end{array}$ & 1 & 2 & 3 & 4 & 5 \\
\hline 15 & $\begin{array}{l}\text { Nos hace saber que nos basemos en el razonamiento y en la } \\
\text { evidencia para resolver los problemas. }\end{array}$ & 1 & 2 & 3 & 4 & 5 \\
\hline 16 & $\begin{array}{l}\text { Trata de que obtengamos lo que deseamos a cambio de nuestra } \\
\text { cooperación. }\end{array}$ & 1 & 2 & 3 & 4 & 5 \\
\hline
\end{tabular}




\begin{tabular}{|c|c|c|c|c|c|c|}
\hline 17 & $\begin{array}{l}\text { Esta dispuesto a instruirnos o enseñarnos siempre que lo } \\
\text { necesitemos. }\end{array}$ & 1 & 2 & 3 & 4 & 5 \\
\hline 18 & Evita hacer cambios mientras las cosas marchan bien. & 1 & 2 & 3 & 4 & 5 \\
\hline 19 & Nos da charlas para motivarnos. & 1 & 2 & 3 & 4 & 5 \\
\hline 20 & Evita tomar decisiones. & 1 & 2 & 3 & 4 & 5 \\
\hline 21 & Cuenta con nuestro respeto. & 1 & 2 & 3 & 4 & 5 \\
\hline 22 & Potencia nuestra motivación de éxito. & 1 & 2 & 3 & 4 & 5 \\
\hline 23 & $\begin{array}{l}\text { Trata de que veamos los problemas como una oportunidad para } \\
\text { aprender. }\end{array}$ & 1 & 2 & 3 & 4 & 5 \\
\hline 24 & Trata de desarrollar nuevas formas para motivarnos. & 1 & 2 & 3 & 4 & 5 \\
\hline 25 & Nos hace pensar sobre los viejos problemas de forma nueva. & 1 & 2 & 3 & 4 & 5 \\
\hline 26 & $\begin{array}{l}\text { Nos deja que sigamos haciendo nuestro trabajo como siempre lo } \\
\text { hemos hecho, a menos de que sea introducir algún cambio. }\end{array}$ & 1 & 2 & 3 & 4 & 5 \\
\hline 27 & Es difícil de encontrarlo cuando surge un problema. & 1 & 2 & 3 & 4 & 5 \\
\hline 28 & $\begin{array}{l}\text { Impulsa a la utilización de la inteligencia para superar los } \\
\text { obstáculos. }\end{array}$ & 1 & 2 & 3 & 4 & 5 \\
\hline 29 & $\begin{array}{l}\text { pide que fundamentemos nuestras opiniones } \\
\text { con argumentos sólidos. }\end{array}$ & 1 & 2 & 3 & 4 & 5 \\
\hline 30 & $\begin{array}{l}\text { Nos da nuevas formas de enfocar los problemas que antes les } \\
\text { resultaban desconcertantes. }\end{array}$ & 1 & 2 & 3 & 4 & 5 \\
\hline 31 & Evita decirnos como se tienen que hacer las cosas. & 1 & 2 & 3 & 4 & 5 \\
\hline 32 & Es probable que esté ausente cuando se le necesita. & 1 & 2 & 3 & 4 & 5 \\
\hline 33 & Tenemos plena confianza en él. & 1 & 2 & 3 & 4 & 5 \\
\hline 34 & Confiamos en su capacidad para superar cualquier obstáculo. & 1 & 2 & 3 & 4 & 5 \\
\hline
\end{tabular}




\section{Anexo 3:}

\section{Cuestionario de Satisfacción S21/26 (Domínguez, Calderón \& Arroyo, 2016)}

CUESTIONARIO 1 (SL). Indicaciones: A continuación, encontrará 20 enunciados, los cuales deberá leer cuidadosamente y marcar con una " $\mathrm{X}$ " en el casillero que corresponda a su grado de acuerdo con la afirmación, dependiendo de su situación actual dentro del ámbito de trabajo. Responda de manera sincera a cada una de ellas.

\begin{tabular}{|c|c|c|c|c|}
\hline 0 & 1 & 2 & 3 & 4 \\
\hline $\begin{array}{c}\text { TOTALMENTE EN } \\
\text { DESACUERDO }\end{array}$ & DESACUERDO & $\begin{array}{c}\text { NI ACUERDO, } \\
\text { NI EN } \\
\text { DESACUERDO }\end{array}$ & DE ACUERDO & $\begin{array}{c}\text { TOTALMENTE DE } \\
\text { ACUERDO }\end{array}$ \\
\hline
\end{tabular}

\begin{tabular}{|c|c|c|c|c|c|c|}
\hline 1 & Me gusta mi trabajo. & 1 & 2 & 3 & 4 & 5 \\
\hline 2 & $\begin{array}{l}\text { Estoy satisfecho con las posibilidades que me da mi trabajo de hacer } \\
\text { las cosas en las que yo destaco. }\end{array}$ & 1 & 2 & 3 & 4 & 5 \\
\hline 3 & $\begin{array}{l}\text { Estoy satisfecho con mi trabajo porque me permite hacer cosas que } \\
\text { me gustan. }\end{array}$ & 1 & 2 & 3 & 4 & 5 \\
\hline 4 & Mi salario me satisface. & 1 & 2 & 3 & 4 & 5 \\
\hline 5 & Estoy satisfecho con la cantidad de trabajo que me exigen. & 1 & 2 & 3 & 4 & 5 \\
\hline 6 & Estoy satisfecho de la formación que me da la empresa. & 1 & 2 & 3 & 4 & 5 \\
\hline 7 & Estoy satisfecho de mis relaciones con mis jefes. & 1 & 2 & 3 & 4 & 5 \\
\hline 8 & La supervisión que recibo es satisfactoria. & 1 & 2 & 3 & 4 & 5 \\
\hline 9 & Estoy a gusto de cómo mi empresa cumple las leyes laborales. & 1 & 2 & 3 & 4 & 5 \\
\hline 10 & Estoy a gusto con la atención y frecuencia con la que me dirigen. & 1 & 2 & 3 & 4 & 5 \\
\hline 11 & $\begin{array}{l}\text { Estoy satisfecho de mi grado de participación en las decisiones de mi } \\
\text { departamento o sección. }\end{array}$ & 1 & 2 & 3 & 4 & 5 \\
\hline 12 & forma en que mis superiores juzgan mi tarea. & 1 & 2 & 3 & 4 & 5 \\
\hline 13 & Mi empresa me trata con buena justicia e igualdad. & 1 & 2 & 3 & 4 & 5 \\
\hline 14 & Estoy contento de apoyo que recibo de mis superiores. & 1 & 2 & 3 & 4 & 5 \\
\hline 15 & $\begin{array}{l}\text { Me satisface mi actual grado de participación en las decisiones de mi } \\
\text { grupo de trabajo. }\end{array}$ & 1 & 2 & 3 & 4 & 5 \\
\hline 16 & Estoy satisfecho del trabajo que realizo con mis compañeros. & 1 & 2 & 3 & 4 & 5 \\
\hline 17 & Estoy satisfecho de los incentivos que me dan. & 1 & 2 & 3 & 4 & 5 \\
\hline 18 & $\begin{array}{l}\text { Los medios materiales que tengo para hacer mi trabajo son adecuados } \\
\text { y satisfactorios. }\end{array}$ & 1 & 2 & 3 & 4 & 5 \\
\hline 19 & Estoy contento del nivel de calidad que obtenemos. & 1 & 2 & 3 & 4 & 5 \\
\hline 20 & Estoy satisfecho del ritmo al que tengo que hacer mi tarea. & 1 & 2 & 3 & 4 & 5 \\
\hline
\end{tabular}

